\title{
Cambio tecnológico y sus efectos en el mercado de trabajo: una revisión analítica ${ }^{1}$
}

\section{Technologic change and its effects on the labor market: an analytic review}

\author{
Luis Huesca-Reynoso* \\ David Castro-Lugo** \\ Reyna E. Rodríguez-PÉREZ*
}

\begin{abstract}
We expose the recent literature and the relevant variables in the analysis of technologic change, as well as their links with human capital, the labor market and the recent research lines in this theme, in the environmental sphere and in Mexico. On the one side, the evidence indicates the absence of an accurate explanation on the nature of relation between technical change and its heterogeneous effect on the laborers; and on the other, that most researches have not used in a proper manner variables that approach the hypothesis of biased technical change in determining the effect of technologies on wages for different attributes.
\end{abstract}

Keywords: technical change, labor market, wages, qualified and non-qualified labor.

\begin{abstract}
Resumen
Se expone la literatura reciente y las variables relevantes en el análisis del cambio tecnológico, así como su vínculo con el capital humano, el mercado de trabajo y las líneas de investigación recientes en esta área temática, en el ámbito internacional y en México. Por un lado, la evidencia indica la ausencia de una explicación precisa sobre la naturaleza de la relación entre el cambio técnico y su efecto heterogéneo en los trabajadores; y por otro, que la mayoría de las investigaciones no han utilizado propiamente variables que aproximen la hipótesis de cambio técnico sesgado en la determinación del efecto de las tecnologías en los salarios para diferentes atributos.
\end{abstract}

Palabras clave: cambio técnico, mercado laboral, salarios, trabajo calificado y no calificado.

\footnotetext{
${ }^{1}$ Los autores agradecen el apoyo económico otorgado por el Conacyt para la realización de esta investigación, que es parte del proyecto Ciencia básica (SEP 2008-01-106684). Asimismo reconocen los comentarios de los evaluadores anónimos.

* Centro de Investigación en Alimentación y Desarrollo, A.C., México. Correos-e: Ihuesca@ciad.mx, reyna@tudiantes.ciad.mx.

** Universidad Autónoma de Coahuila, México. Correo-e: david.castro@uadec.edu.mx.
} 


\section{Introducción}

El cambio tecnológico ha impuesto, en el ámbito mundial en las últimas tres décadas, la pauta en una creciente demanda relativa de trabajadores con mayor formación y habilidades, lo que explica más de una tercera parte de los cambios producidos en la estructura laboral de los países (Autor et al., 2003, 2008). En Estados Unidos llega a explicar entre 30 y $40 \%$ de dichos cambios, y de acuerdo con evidencia reciente, se asume que las habilidades cognitivas de los trabajadores pasan a jugar un papel central en la contratación por parte de las empresas (Bowles et al., 2001). La creciente utilización de sistemas informáticos y de computadoras en los centros de trabajo ha sido esencial en este proceso (Acemoglu, 2002; Autor et al., 2003).

Es bien sabido que a partir de la Revolución industrial las tecnologías han venido sustituyendo al factor trabajo calificado, sin embargo, recientemente el cambio técnico viene a complementarlo. Al respecto, algunas investigaciones clasifican las actividades del trabajo en tareas rutinarias y no rutinarias, estableciendo que la tecnología sí puede sustituir a las primeras, las cuales solían realizar los trabajadores. Las labores rutinarias son todas las actividades que pueden desarrollar maquinas o que siguen reglas de programación; en tanto que las no rutinarias son aquéllas en las que las reglas no están suficientemente bien establecidas y no han podido ser codificadas por computadoras o ejecutadas de manera sistemática. La temática de la discusión previa es parte del cometido de este trabajo.

También se observa que, temporalmente, el aumento de la desigualdad en los ingresos de los trabajadores se han concentrado en la década de 1980, cuando se produjeron cambios relevantes en la microinformática y en los procesos de trabajo. Aquí la hipótesis del cambio técnico sesgado es un elemento relevante en el aumento de la desigualdad entre los distintos grupos de trabajadores calificados y los no calificados; por otro lado, la complementariedad de las tecnologías permite mayor adaptación en las generaciones jóvenes, sin embargo, los individuos con mayor edad siguen obteniendo mayores premios salariales por su experiencia.

Este trabajo tiene el objetivo de exponer la literatura reciente y las variables relevantes en el análisis del cambio técnico y su vínculo con el capital humano y el mercado de trabajo; así como presentar las hipótesis y exponer las líneas de investigación recientes en esta área. El trabajo presenta el siguiente orden. La primera parte describe la evolución reciente del cambio tecnológico y sus implicaciones, la hipótesis del cambio técnico sesgado y su relación con la demanda laboral. La segunda parte muestra la evidencia empírica en el caso de México, la cual se divide en tres secciones: 1) los efectos de la tecnología de acuerdo con distintos 
atributos de los individuos, como la educación, el género y la edad; 2) el análisis económico por sectores y ramas, y 3) el contexto regional. En el tercer apartado se analiza en conjunto el efecto del cambio tecnológico vinculado con los atributos previamente analizados. Por último, se presentan las conclusiones.

\section{Revisión teórica y empírica: implicaciones y contrastes}

Las siguientes preguntas sirven de guía en la revisión que este trabajo realiza. ¿Cuáles son los efectos de la tecnología en el mercado laboral?, ¿cómo afecta la distribución del salario e ingreso?, ¿es la tecnología responsable del cambio en la estructura salarial observada en muchos países en las últimas décadas? Estas son algunas preguntas que autores como Acemoglu (2002) se plantean, y expresan bien la preocupación de los economistas laborales y de los estudiosos del tema laboral en general.

A partir de una serie de estudios sobre el tema en diferentes países, se puede establecer que en su mayoría encuentran que existe una relación directa entre la adopción de tecnología y la demanda de mano de obra calificada, basados en la evidencia empírica entre sectores, entre empresas y al interior de las mismas; hecho que permite concluir a los estudiosos que el cambio tecnológico es sesgado a favor de los trabajadores más calificados. Sin embargo, no siempre se establece cuál es la causa o naturaleza de esta relación, así como los elementos que determinan la incorporación de tecnología en las actividades económicas. Por ello, el primer aspecto a analizar en este documento es precisamente ése.

Existen diferentes modelos que intentan establecer esta relación, entre los cuales destacamos el de Autor et al. (2003) por su claridad y racionalidad, motivo por el cual lo utilizamos como base en esta sección, y a partir de él hacemos las consideraciones correspondientes.

Este modelo comienza con la pregunta: ¿qué tareas se pueden realizar con una computadora? ${ }^{2}$ Evidentemente la respuesta no es absoluta, sino relativa, dado que dependería de la era tecnológica a la que se haga referencia: no es lo mismo computadoras de principios del siglo xx que las que existen un siglo después, pues la capacidad de procesamiento de información aumentó exponencialmente; así, hoy en día las computadoras pueden realizar actividades mucho más variadas y en mucho menos tiempo que unas décadas atrás. Por otra parte, aun cuando la tecnología puede hacer más tareas que antes, otra pregunta pertinente es: ¿cuál es el costo de esta tecnología? No solamente es relevante que exista, sino además que esté accesible; al respecto, junto al aumento de la capacidad de

${ }^{2}$ Considerando ésta como una personificación de la tecnología. 
procesamiento de las computadoras y su diversidad de aplicaciones, éstas han presentado una reducción sustancial en su costo, lo que puede interpretarse como la posibilidad de que las empresas que pueden acceder a ella represente un número cada vez mayor.

Este avance tecnológico, ¿cómo afecta la composición del trabajo? La respuesta depende de si la computadora complementa o sustituye a la actividad humana. Para responder esta pregunta, Autor et al. (2003) clasifican el trabajo en tareas rutinarias y no rutinarias, donde las primeras constituyen todas las actividades que pueden desarrollar máquinas o siguiendo reglas de programación explícitas, mientras que las segundas serían aquéllas en las cuales las reglas no están suficientemente bien establecidas para ser codificadas por computadoras o ejecutadas por máquinas. Dada esta clasificación, es posible establecer que la tecnología puede sustituir las tareas rutinarias realizadas por los trabajadores.

Este hecho en sí no constituye ningún punto relevante, pues a partir de la Revolución industrial se ha dado que las máquinas sustituyan al hombre. Así, ¿qué es lo destacable en las últimas décadas y que tiene efectos en el mercado laboral? En lo fundamental podemos destacar dos elementos. El primero, ya mencionado, es la relativa reducción del precio de la tecnología, conforme es más barata, existe un mayor incentivo para que se le incorpore al proceso productivo, dado que la decisión de introducir tecnología respondería a un criterio de minimización de costos; por tanto, que las computadoras sustituyan tareas rutinarias estará determinada por los precios relativos de los factores sustituibles; en este sentido, cuanto mayor sea la reducción del costo tecnológico, manteniendo el costo de mano de obra rutinaria sin cambios, mayor será el incentivo para incrementar el uso de tecnología. El segundo elemento a destacar es que la era de la computación y la informática han permitido incorporar un mayor número de actividades en el concepto de tareas rutinarias, lo cual ha venido a ampliar y acelerar el proceso de sustitución de mano de obra por maquinaria, mismo que se refleja en los diferentes sectores económicos.

Mientras la computadora sustituye el trabajo rutinario, ésta puede tener un carácter complementario con las tareas no rutinarias, por ejemplo, aunque con la computadora se pueda hacer el procesamiento de una cantidad impresionante de información en muy poco tiempo, no es capaz de realizar estudios científicos, aunque sí permite aumentar la productividad. Es en este sentido que se puede establecer la complementariedad entre la tecnología y el trabajo no rutinario.

A partir de lo anterior, se está en condiciones de responder acerca de la relación y efectos que parece tener el cambio tecnológico, personificado en la utilización de la computadora y la informática, sobre el mercado laboral, y específicamente en la estructura ocupacional y/o desigualdad 
salarial. La deducción que se puede establecer a partir de este comportamiento es que el efecto del cambio tecnológico en el mercado laboral no es neutral en las distintas ocupaciones o dotaciones de mano de obra.

Desagregando la conclusión anterior, una primera implicación sería que la demanda de trabajadores con actividades rutinarias, mismos que puede definirse como no calificados, ${ }^{3}$ se reduciría sustancialmente porque este tipo de trabajo se sustituye por maquinaria, mientras que debido a la complementariedad entre trabajo no rutinario -el cual se puede definir como calificado- y el uso de tecnología, la demanda de este tipo de mano de obra aumentaría.

Una segunda implicación sería sobre las remuneraciones salariales. Para evitar la sustitución de trabajo rutinario por maquinaria, es necesario que el costo de la mano de obra rutinaria -expresado en unidades de eficiencia- no supere al del capital, mismo que en las últimas décadas ha mostrado una reducción permanente, por tanto, el costo laboral del trabajo rutinario debe bajar. Por otra parte, la mayor utilización de computadoras y la complementariedad de éstas con las tareas no rutinarias, incidirían en la productividad de la mano de obra calificada, y como consecuencia, el salario de estos trabajadores aumentaría, dando como resultado un incremento en la desigualdad salarial.

Las ideas presentadas hasta ahora establecen con claridad la dinámica del cambio técnico, el cual viene a sustituir a las tareas rutinarias y el nivel de sustitución estará determinado por el costo relativo de la tecnología y la mano de obra que realiza estas actividades. Por otra parte, las computadoras complementan las tareas no rutinarias incrementando la productividad, hecho que permite establecer la existencia de un cambio técnico sesgado.

Uno de los mayores problemas a los que se enfrentan quienes estudian la hipótesis de cambio técnico sesgado (HCTS) es cómo capturar este efecto. Para su estudio, Card y DiNardo (2002) sugieren diferentes alternativas: establecen que ésta puede ser probada utilizando tanto información sobre salarios como oferta laboral en términos relativos para diferentes grupos, mismos que pueden clasificarse por nivel educativo, edad, sexo, entre otros indicadores no menos importantes.

También hay que considerar que el análisis se puede llevar a cabo en distintos niveles de agregación. Si es a nivel agregado, el objetivo sería establecer la relación entre remuneraciones relativas y tecnología. Aquí el reto es definir un indicador de este último concepto. Una forma práctica y comúnmente utilizada, aunque no exenta de cuestionamientos, es tomar

\footnotetext{
${ }^{3}$ Se define así porque una proporción importante de las ocupaciones rutinarias demandan trabajo con baja calificación.
} 
como indicador la presencia de la computadora personal. Así el contraste de la нстs consistiría en observar el comportamiento de la disparidad salarial con el grado de utilización o generalización de la computadora en las actividades económicas. ${ }^{4}$ Aun cuando puede resultar simple el razonamiento, puede ser sumamente complicado determinar la fase de desarrollo tecnológico y especialmente la magnitud de utilización de esta tecnología en la economía.

Para el caso de grupos específicos, es posible considerar los argumentos planteados por Juhn et al. (1991, 1993) y Autor et al. (1998). Estos trabajos sostienen que las personas que están más relacionadas con la utilización de computadoras tienen mayor capacidad para aprovechar la innovación y aumentar la productividad, hecho que se reflejará en mayores posibilidades de incorporación al mercado laboral dentro de los nuevos procesos de producción. Card y DiNardo (2002) definen esto como la versión complementariedad entre computadoras y habilidades (computeruse-skill complementary) de la HCTs; mientras que Juhn et al. (1993) asumen que los cambios tecnológicos han incrementado la productividad relativa de los trabajadores más calificados, lo que ha generado una expansión de las diferencias salariales entre grupos, situación que se define como hipótesis de aumento del premio a las habilidades (rising-skill-price).

A partir de lo anterior, se pueden definir dos formas básicas mediante las cuales se aborda la hipótesis de cambio técnico. La primera medida, derivada del enfoque de la complementariedad, estaría relacionada con los cambios generados en la estructura ocupacional de los trabajadores que realizan uno y otro tipo de tareas, y se podrían mostrar evidencias mediante una menor presencia de trabajadores no calificados en la estructura laboral, o en su defecto, que la tasa de crecimiento de este tipo de empleo es menor en términos relativos, mientras que el caso contrario se presentaría para los trabajadores que desarrollan tareas no rutinarias. Una fuente alternativa asociada a la estructura laboral es considerar la proporción de trabajadores que utilizan computadoras o tecnologías de información en su actividad.

Una segunda opción es vía las remuneraciones salariales, la cual aporta el enfoque del premio. Partiendo de una oferta laboral sin cambios, la reducción en la demanda de mano de obra no calificada presionaría hacia abajo los salarios, o en su defecto crecerían a un ritmo menor que el promedio, mientras que los trabajadores calificados verían incrementar su demanda, producto del aumento en la productividad, y sin variaciones en la oferta, el salario relativo se elevaría frente a las actividades rutinarias.

\footnotetext{
${ }^{4}$ Ésta puede considerarse en el sentido más amplio, es decir, incluyendo la relacionada con las actividades de Internet e informática.
} 
Una forma alternativa es analizar el comportamiento de la desigualdad salarial, suponiendo que dicho comportamiento es resultado de una reducción salarial en la parte baja de la distribución y un aumento en la parte alta.

¿Se puede considerar indistinto utilizar uno u otro enfoque? La respuesta es no, y la decisión de tomar uno u otro indicador dependerá del objetivo que se persiga, aunque si el propósito es estudiar el efecto de la tecnología en el mercado laboral, lo deseable es utilizar ambos, dado que pueden ser complementarios, pero se debe reconocer que una de las mayores preocupaciones es determinar en qué medida el cambio técnico incide en la desigualdad salarial.

Posiblemente éste sea el motivo, además de la disponibilidad de información, por el cual existe una mayor cantidad de estudios que emplean este enfoque frente a la opción de la estructura; sin embargo, debe reconocerse que el contraste de la нстs vía ingresos, al menos para el caso de los países en desarrollo, presenta mayores limitaciones, dado que el comportamiento absoluto y relativo de los salarios puede ser compatible con otras hipótesis alternativas a la HCTs, como puede ser la reducción del salario mínimo real para el caso de los trabajadores no calificados y de los factores institucionales en general, y la existencia de rigidez de la oferta laboral en trabajadores calificados, así como elementos asociados con modificaciones en la política comercial.

Lo anterior permite establecer que el enfoque del premio a las habilidades puede estar reflejando transformaciones en el mercado laboral atribuibles a elementos distintos al cambio técnico, como los mencionados antes, y en su caso pueden estar jugando un papel complementario, especialmente en países caracterizados como economías cerradas y que a principios de los ochenta iniciaron un proceso de apertura comercial, que coincide con la expansión de la tecnología computacional, como es el caso de la economía mexicana.

El proceso de apertura comercial se expresó en la reducción arancelaria y en la eliminación de cuotas de importación, así como de otros instrumentos de protección, situación que permitió reducir el costo de importación de tecnología y abaratar, aún más, el precio de las computadoras incentivando adicionalmente la sustitución de trabajo rutinario por tecnología. Así, la apertura comercial pudo jugar el papel de catalizador en el proceso de adopción tecnológica de las empresas (Meza, 2003). ${ }^{5}$

Un elemento adicional que debe tomarse en cuenta en la exploración de la нстs para el caso de México, es que durante el proceso de reestruc-

${ }^{5}$ Junto con la reducción de la protección comercial, durante los primeros años de la década de los noventa el peso presentó un nivel importante de sobrevaluación. 
turación económica, se llevaron a cabo diferentes reformas constitucionales, entre las cuales destaca la modificación en las disposiciones en materia de inversión extrajera directa (IED). Este elemento y el esquema maquilador y posteriormente la firma del TLCAN, permitieron recibir volúmenes importantes de IED, misma que pudo incorporar más tecnología a los procesos productivos, lo que tuvo efectos en la demanda de mano de obra complementaria y en los diferenciales de salarios (Hanson, 2003).

A partir de lo anterior se puede establecer que la apertura de la economía (en materia comercial y de inversión) y la política monetaria llevada a cabo en los primeros ańos de los noventa, actuaron a favor de un cambio tecnológico sesgado.

Por otra parte, es posible destacar que hay tres elementos que apuntarían en la dirección opuesta. El primero estaría relacionado con la existencia de mercados financieros poco desarrollados y elevados costos de intermediación, lo cual constituye una limitante para la sustitución de tareas rutinarias por tecnología computacional, por los elevados y escasos recursos crediticios, aspecto que afecta especialmente a las empresas micro y pequeñas. El segundo elemento lo podemos ubicar en el concepto de factores institucionales, donde se puede mencionar la desregulación y flexibilidad del mercado laboral ${ }^{6}$ y la pérdida de salario real, sobre todo de los trabajadores menos calificados. El tercer elemento sería la volatilidad de los mercados de bienes, por los fuertes cambios en la demanda. En estas condiciones las empresas requieren gran flexibilidad en su estructura de costos, y a partir del segundo punto, la mano de obra presenta más ventajas frente a la tecnología.

De acuerdo con el modelo y con los criterios de decisión descritos, esto pudo incidir desfavorablemente en la incorporación de tecnología, dado que la reducción de costo y flexibilidad laboral, así como la volatilidad de la demanda de bienes, hacen menos atractiva la sustitución de tareas rutinarias por computadoras.

A partir de lo presentado hasta ahora, es posible sostener, en primer término, que el incremento de la desigualdad salarial entre trabajadores calificados y no calificados, como evidencia de la нстs, puede ser compatible con otras hipótesis; y segundo, que para el caso de los países en desarrollo, y para México en particular, durante el periodo de estudio del cambio técnico, éstos coincidieron con una serie de elementos que muestran la existencia de conectividades donde unos pueden actuar como catalizadores del proceso y otros en dirección opuesta.

Lo anterior indicaría que hacer un contraste empírico sobre la нстs debe llevarse a cabo con detalle, sometiendo la información a diferentes

${ }^{6}$ Ésta puede incluir modificaciones legales o reales. 
criterios que permitan establecer, con la mayor consistencia posible, que las transformaciones presentadas en el mercado laboral, en especial en lo relativo a disparidad salarial, son producto del cambio técnico sesgado y no de otro factor.

Como se mencionó, el contraste de la нстs se puede analizar en subgrupos, así es posible ver si la utilización de la tecnología computacional está más relacionada con hombres o con mujeres, y si este comportamiento se materializa en alteraciones en su estructura y/o en diferencias salariales. Esto se puede observar para toda la población ocupada por género o desagregando por subgrupos de edad o niveles educativos. Si el estudio se realiza al interior de las empresas, se apreciaría la evolución del contenido tecnológico en la misma, la composición de la estructura ocupacional y el desempeńo de las remuneraciones de los trabajadores en tareas rutinarias y no rutinarias; estos elementos permitirían contrastar la HCTS, considerando tanto el factor de complementariedad como de premio a las habilidades.

Dada la existencia de heterogeneidad en las actividades económicas en materia de requerimientos de tecnología y mano de obra, así como la presencia de distintas potencialidades para su incorporación, estos elementos abren el espacio para explorar evidencias sobre la HCTs analizando el comportamiento de los distintos sectores o actividades.

Una de las implicaciones del modelo y su dinámica de funcionamiento es que las industrias teóricamente intensivas en trabajo rutinario, con la reducción de los precios de la tecnología en computación, tendrían mayores incentivos para sustituir trabajadores rutinarios por tecnología. Así, estas industrias serías las que llevarían a cabo una mayor incorporación. Inicialmente los sectores de alta tecnología deberían reflejar una mayor dotación de mano de obra calificada, pero observando la evolución en el tiempo, específicamente durante los ochenta y noventa, el crecimiento relativo de las industrias intensivas en trabajo no calificado debería incorporar mayor proporción de trabajo no rutinario.

Existe evidencia empírica que sostiene que hay grupos que son más compatibles con las computadoras, suelen ser más productivos y sus salarios son más altos respecto a los que no tienen dicha compatibilidad, este argumento lo sustentan Autor et al. (1998), quienes asumen que los grupos que tienen más probabilidades de utilizar el equipo computacional tienen mayores salarios en el mercado laboral. Del mismo modo, los estudios sobre esta temática con una orientación regional constituyen una opción real e interesante, considerando que los efectos generados por la globalización imprimen un efecto heterogéneo en el territorio de los países en desarrollo (Hanson, 2003). Esto permite establecer los elementos generales sobre los cuales se pueden evaluar o discutir los estudios que 
exploran la existencia de cambio técnico sesgado y determinar el estado del arte en este tema en México, actividad que se realiza en el siguiente apartado.

El análisis de la evidencia empírica respecto a la HCTs para el caso de México, se puede organizar en torno a seis preguntas clave: ¿¿de qué manera se relaciona el nivel educativo y los salarios con la adaptación e implementación de la tecnología?, ¿los efectos del cambio tecnológico en los salarios son iguales para las mujeres que para los hombres?, ¿qué papel juega la edad en el manejo y adaptación de las tecnologías?, ¿se refleja en los salarios de los más jóvenes?, ¿cómo se comporta la incorporación de la tecnología, el empleo y los salarios en diferentes sectores y ramas económicas? y, ¿se puede considerar que la tecnología tiene el mismo efecto en todas las regiones de México? La respuesta a estas preguntas nos permitirá determinar el nivel de análisis sobre esta temática y acercarnos a la comprensión del efecto que ha tenido la HCTs en el mercado laboral mexicano.

\section{Evidencia empírica en el caso de México}

\subsection{Efectos de la tecnología en individuos con distintos atributos}

\subsubsection{Relación entre educación e implementación de tecnología}

Las comparaciones de uso de computadora entre niveles educativos revela un importante elemento para explicar la desigualdad salarial y el comportamiento de la estructura ocupacional. Card y DiNardo (2002) muestran que los grupos que tienen educación básica, cuentan con más probabilidades de utilizar un ordenador en el trabajo, en comparación con quienes abandonan la escuela; y por otra parte, los que son graduados universitarios tienen aproximadamente el doble de posibilidad de utilizar una computadora en relación con los que sólo tienen educación secundaria. Lo anterior muestra que existe más complementariedad entre las personas con mayor grado de educación y la tecnología en relación con quienes tienen bajo nivel de estudios.

Parece plausible que la revolución informática conduciría a un aumento de la demanda relativa de los graduados universitarios con más conocimientos técnicos, como los ingenieros y los científicos, especialmente en el decenio de 1980, cuando se introdujo por primera vez la microinformática. A raíz de esto, la brecha salarial de los trabajadores que tenían secundaria en relación con los que tenían estudios universitarios se amplió rápidamente. $\mathrm{Al}$ respecto, Card y DiNardo (2002) argumentan que los salarios relativos de los graduados universitarios que se encuentran en los 
campos más estrechamente relacionados con las computadoras, informática e ingeniería eléctrica han aumentado en relación con aquéllos que están en las áreas de humanidades y ciencias sociales; asimismo sostienen que el desarrollo de las tecnologías de información y comunicación (TIC) de fines del decenio de 1990 se asoció a un aumento de los salarios de los graduados con habilidades relacionadas a éstas.

El manejo y adaptación de los individuos a la tecnología está sesgando la demanda de trabajo. Oliver et al. (2001), en su estudio para España, encuentran evidencia empírica que sugiere que el cambio tecnológico está sesgando la demanda de trabajo hacia aquellos individuos con mayor educación, de manera que la prima salarial que éstos obtendrían tendería a crecer. Lo anterior muestra que en el ámbito internacional hay elementos para sostener que la desigualdad salarial se debe al cambio tecnológico acaecido en las últimas décadas.

La economía mexicana no está exenta de estos cambios y ha experimentado transformaciones importantes en su modelo de desarrollo, al pasar de una economía cerrada con una importante participación del Estado y regulación, a un modelo con fuerte orientación hacia el exterior, donde participan empresas extranjeras y nacionales que tienen mayores requerimientos de mano de obra calificada. Sin embargo, a partir de 1980 los salarios en México se estancaron y su dispersión aumentó. En este contexto es importante preguntarnos, ¿¿de qué manera se relaciona el nivel educativo y los salarios con la adaptación e implementación de la tecnología?

Hanson (2003) sostiene que las reformas económicas en México parecen haber incrementado la demanda de trabajo calificado, y que estos cambios han resultado en un incremento de la dispersión salarial. Por su parte, Bracho y Zamudio (1994) y Zamudio (1995), utilizando la Encuesta Nacional de Ingreso y Gasto de los Hogares (ENIGH) de 1989, muestran que la tasa de rentabilidad por cada año educativo cursado es de $11.7 \%$, y en el caso de la educación superior se muestra una mayor utilidad en relación con la media superior. Estos resultados coinciden con los obtenidos por Barceinas (2003) en el periodo 1994-1996.

En este mismo contexto, Urciaga (2002) presenta un trabajo donde calcula la rentabilidad de la educación por medio del método extendido y elaborado, para lo cual utiliza la base de datos de la ENIGH de 1998. Sus resultados indican que en promedio una persona que cuenta con educación primaria completa le permite recibir $47 \%$ en salario en relación con quien no tiene estudios, y para los egresados de secundaria el porcentaje es de $80 \%$, en preparatoria de $128 \%$ y en el caso de los estudios universitarios el nivel es de $184 \%$. Lo que muestra el incremento de los rendimientos educativos con el nivel de instrucción, pero como el estudio sólo 
analiza un ańo, no es posible identificar el comportamiento de la brecha en los rendimientos educativos a lo largo del tiempo.

Igualmente, Huesca (2004 y 2005) realizó un análisis de los rendimientos de la educación con los datos de la ENIGH en el periodo 1984-2000 y con la Encuesta Nacional de Empleo Urbano (ENEU) 1990-2002, respectivamente, donde calcula que la inversión educativa tiene en promedio $11 \%$ de retorno por cada año educativo obtenido en el primer estudio, y que incluso el sector informal laboral ha mostrado que los rendimientos han ido aumentando en el periodo analizado en el segundo trabajo de investigación.

Recientemente Villareal (2008), con datos de la ENEu para los años 1987-2004, obtiene las mayores tasas educativas en el nivel superior, respecto de grados menores. Según la autora, una posible explicación a este fenómeno ha sido el alza en la demanda de trabajadores calificados durante el periodo 1980-1990, debido al crecimiento de las innovaciones tecnológicas intensivas en conocimiento a favor de los trabajadores con mayores niveles educativos. En esta tónica, Urciaga y Almendarez (2008) estimaron rendimientos en la frontera norte del país con la ENEU a una tasa de $10 \%$, encontraron que los mejores salarios se pagan en las ciudades ubicadas en la línea de frontera y las de mayor desarrollo relativo, mientras que las más alejadas de dicha zona reflejan los menores ingresos. Las razones que presentan son la mayor especialización de la mano de obra que contrata la industria maquiladora con estudios técnicos especializados.

En relación con las referencias comentadas, se puede mencionar que efectivamente los resultados indican un incremento en los rendimientos educativos, pero los autores no utilizan variables que reflejen el cambio tecnológico, aun cuando se puede argumentar que los aumentos salariales tuvieron lugar en la década de gran auge en el crecimiento y la adaptación de la tecnología en todo el mundo y en México, aunque estos autores sí lo consideran como un elemento relevante en la explicación. También existen otros factores distintos al cambio tecnológico, compatibles con el comportamiento del rendimiento educativo.

Romero y Puyana (2003) aportan un elemento adicional que contrasta los estudios anteriores. El trabajo realiza un análisis de la viabilidad de invertir en educación en el periodo 1980-2000, utilizando 73 ramas con los datos de la Clasificación Industrial Internacional. Los resultados indican que la educación de la fuerza de trabajo mejoró a partir de 1991, sin embargo, este considerable aumento en el empleo calificado no obedece a un cambio tecnológico, sino que constituye básicamente una forma de conseguir empleo en el sector moderno. Por lo que mayores inversiones en capital humano en el actual crecimiento no se traduce en mayores niveles de eficiencia, y que puede incluso traducirse en una dis- 
minución de la dispersión salarial pero a la baja, reduciendo los salarios de los más calificados.

\subsubsection{Efectos de la tecnología en el empleo por género}

En la cultura occidental, manejar la tecnología más reciente es una actividad altamente valorada y mitificada. Los saberes tecnológicos son reconocidos y constituyen una importante fuente de poder para la humanidad. Sin embargo, Arango (2004) menciona que las mujeres aparecen como poco aptas para los propósitos tecnológicos, pero esta percepción no coincide con lo que mencionan Card y DiNardo (2002), quienes aseveran que las mujeres son más propensas que los hombres a usar la computadora en los centros de trabajo, en la medida en que la complementariedad con la tecnología basada en las computadoras se mide con la tasa de utilización de las mismas; comportamiento que ha presionado a la alza los salarios de las mujeres en relación con los hombres.

Los mismos autores reportan que para Estados Unidos la brecha salarial por género se incrementó en la década de los setenta, sin embargo, durante los ochenta y principios de los noventa se redujo, mientras que en el segundo lustro de los noventa la diferencia se mantuvo estable. Una posible explicación a este fenómeno es que, dado que las mujeres utilizan relativamente más ordenadores en los centros de trabajo que los hombres, se podría argumentar que la disminución de la brecha salarial por género es coherente con la habilidad del uso de la computadora y su complementariedad con la HTCS. Sin embargo, se sabe que las mujeres que tienen estudios universitarios son menos propensas que los hombres a utilizar la computadora, ya que en este nivel profesional el grado de complejidad en el uso de la tecnología aumenta. Por tanto, al igual que Blau y Kahn (1997), concluyen que un aumento de los salarios de las mujeres en relación con los hombres durante la década de los ochenta, se atribuye a los factores específicos de género.

Para el caso de México, ¿̇los efectos del cambio tecnológico son iguales para las mujeres con respecto a los hombres? Evidenciando la experiencia sobre el tema, Barceinas (1999) mide la rentabilidad de la inversión en educación por niveles para hombres y mujeres con datos de la ENIGH 1992, y muestra que los hombres tienen tasas de rentabilidad mayores en los niveles de primaria y superior en relación con las mujeres, aunque el documento hace referencia a elementos que reflejan cambio tecnológico. Aun cuando existen diferencias en 1992, no sabemos cuál fue su comportamiento antes o después de ese año.

Por su parte, Bracho y Zamudio (1994), con datos de la ENIGH de 1989 , presentan coeficientes de rentabilidad por género que no muestran 
diferencias significativas estadísticamente: de 11.9 y 11.6 entre hombres y mujeres, respectivamente. Meza (2001), con base en datos de la ENEU 1988-1998, obtiene que la brecha salarial entre hombres y mujeres se redujo en ese periodo, misma que pudo ser generada por las modificaciones que se presentaron en el mercado de trabajo mexicano por el cambio tecnológico, la mayor competitividad y la apertura comercial.

Por su parte, Martínez y Acevedo (2004) hicieron un estudio basado en la ENIGH del año 2000, en el contexto de una mayor incorporación de las mujeres al mercado laboral. Tal estudio reveló que, en general, gran parte de la brecha salarial se encuentra en las diferencias de calificación de los puestos de trabajo; aunque también, alrededor de $15 \%$ de la discriminación de ingreso se explica por una mayor productividad marginal relativa de la mujer, y que no se refleja en el salario que reciben.

Para la ciudad de Hermosillo, Valenzuela (2002), con la ENEu de 1998, tomó una muestra de universitarios que dividió en dos submuestras, hombres y mujeres; sus resultados reflejan tasas de rentabilidad muy similares para ambos sexos (8.61 y 8.47, respectivamente). En este caso no se puede mencionar que existan diferencias salariales.

Por su parte, Grijalva (2003) realizó un trabajo con datos de una encuesta propia (Encuesta de Empleo, Calificación y Salarios en Hermosillo) para 1995, y sus resultados muestran un promedio en favor de los hombres con un salario promedio de las mujeres $17 \%$ inferior. Su estudio no estima ecuaciones por separado para hombres y mujeres, tampoco interacciona con la variable de sexo. Aun así, indica que las variables relacionadas con la ubicación de las mujeres en determinadas empresas son importantes en la determinación salarial, lo que posiblemente se deba a discriminación por parte de los empleadores.

También para Hermosillo, Rodríguez y Camberos (2007), con datos de la ENEu 2001, analizan la tasa de rentabilidad de la educación por género, sus resultados apuntan a que los hombres ganan en promedio $32 \%$ más que las mujeres. Corrigiendo por sesgo de selección y con el empleo de ecuaciones por separado para hombres y mujeres, detectan en la submuestra de universitarios que las mujeres prefieren ubicarse en carreras de la educación, humanidades y disciplinas artísticas, donde los salarios no son los mejores y existe poco o nulo manejo de las tecnologías. Con la técnica de Oaxaca (1973), detectan que las mujeres obtuvieron en las ingenierías 5\% más de salario, y de esta diferencia, 95\% se debió a productividad y el restante 5\% a discriminación.

Recientemente se encontró una investigación que contrasta con las mencionadas, ya que Villarreal (2008) hizo estimaciones para toda la República Mexicana con la ENEU 1987-2004, sus resultados muestran que en todos los niveles educativos los rendimientos son mayores para el 
grupo de las mujeres en relación con el de los hombres, ${ }^{7}$ una posible causa de esto podría darse porque en México el nivel educativo y la participación de las mujeres se ha incrementado de manera considerable en las últimas décadas debido a las innovaciones tecnológicas intensivas en conocimiento.

A partir de la revisión de los estudios para México, se pueden destacar tres elementos: 1) con excepción de la última referencia, el resto sólo analiza un año, con la limitante que ello implica por la ausencia del efecto longitudinal; 2) los resultados no parecen ser concluyentes, pues mientras los estudios que se apoyan en la ENIGH registran diferencias de ingresos importantes a favor de los hombres, esto no se presenta en los documentos que utiliza la ENEU, lo que parece indicar que la fuente de información incide en los resultados, y 3) los estudios revisados sólo analizan el comportamiento de la brecha salarial o rendimientos educativos, pero no contrastan hipótesis donde exploren las causas de dicho desempeño asociado con el cambio técnico. Desde esta perspectiva, la primera pregunta no se puede responder satisfactoriamente, por lo que es evidente la necesidad de desarrollar investigación que estudie no sólo el comportamiento de la desigualdad salarial por género, sino también su relación con la HCTs.

\subsubsection{La edad y el cambio tecnológico}

En la actualidad se observa que las personas más jóvenes tienen mayor capacidad y adaptación en el manejo y aplicación de la tecnología en relación con los mayores. Una de las posibles explicaciones a este fenómeno es que existe un boom en las Tic debido a la reducción de los costos de las computadoras y otros aparatos electrónicos, como videojuegos, teléfonos celulares, reproductores de música, entre otros, aunado a la popularidad que ha ganado la Internet entre este grupo de personas. Por otro lado, se ha demostrado que entre los mayores existe más complejidad y resistencia al uso y actualización de las tecnologías, ya que este grupo de la población utilizó una computadora a una edad mayor y las innovaciones avanzan tan rápido, que es difícil actualizarse constantemente, lo que ha provocado que cuando los jóvenes se incorporan al mercado laboral, son expertos en hardware y software de cierta complejidad, y por tanto sus salarios son mayores.

Así, un elemento importante a considerar en la utilización de tecnología es la edad. Card y DiNardo (2002) aseveran que la tasa de utilización de uso de computadora aumenta ligeramente entre edades de 20 a 45

\footnotetext{
${ }^{7}$ Comportamiento que confirman Ghiara y Zepeda (2004) para la ciudad de Tijuana.
} 
años y disminuye en mayores de 50, por tanto, el uso de la computadora podría haber reducido los salarios relativos de las personas con más edad al menos en el decenio de 1980. Por otro lado, no se puede dejar de mencionar que las personas que tienen más edad cuentan con mayor experiencia, lo que podría estar aumentando sus salarios, si se toma en cuenta este último elemento.

En años recientes se ha demostrado que los salarios en educación se han diferenciado según el nivel de experiencia de las personas. Durante el periodo 1975-1999, el regreso a la escolaridad era más alto en hombres de mayor edad que en los jóvenes. A partir de la década de los ochenta, los beneficios para los hombres jóvenes se aceleraron rápidamente, con muy pocos cambios para los hombres mayores. Aunque la HCTs no predice las diferencias entre grupos, de la misma hipótesis se puede predecir que el uso de la computadora es más complementario en los jóvenes que en los mayores, debido a que para los primeros es más fácil familiarizarse con ellas porque nacieron en la era de la revolución de las Tic. Un aspecto adicional es que los jóvenes presentan mayor capacidad de adaptación a los nuevos sistemas de producción, caracterizados por ser sumamente cambiantes.

Es posible que la computadora hay inducido un cambio en la eficiencia relativa de los trabajadores jóvenes y mayores con igual o diferentes niveles de educación, dando lugar a cambios en las brechas salariales. Por tanto, el nivel educativo por sí solo no explica el desempeño de la brecha salarial, sino que es necesario explorar otros factores que lo puedan explicar, como puede ser la edad, entre otros elementos.

Por su parte, Card y Lemiux (2001) muestran que en la década de 1960 y principios de 1970, la brecha salarial de quienes tenían estudios de secundaria y con una edad de 45 a 60 ańos, era ligeramente mayor que los que tienen de 26 a 35 años; mientras que después de 1990 la diferencia salarial es mucho mayor a favor de los trabajadores más jóvenes. Los mismos autores demuestran que la tasa de crecimiento de los graduados universitarios ha aumentado en los últimos 40 años, por lo que se podría argumentar que la propagación de las computadoras ha venido a incrementar la productividad de los graduados universitarios; sin embargo, no hay ninguna prueba directa para soportar dicho fenómeno; aunque existe evidencia de que la tecnología pudo haber tenido diferentes efectos según la profesión.

Por otro lado, junto con la educación, la profesión, la edad y el sexo, la quinta dimensión clave de los salarios en Estados Unidos es la experiencia en el mercado laboral. A partir de Mincer (1974), la mayoría de los analistas del mercado laboral han adoptado la hipótesis de que edu- 
cación más experiencia en el mercado laboral son factores que explican los rendimientos.

Como se mencionó, la brecha salarial entre grupos con diferente nivel educativo se amplió a favor de los más jóvenes durante los ochenta y noventa, lo que sugiere que hubo cambios en el diferencial de experiencia de los distintos grupos de educación. Card y DiNardo (2002) mencionan que el uso de la computadora y su complementariedad con la HCTs sugiere que la experiencia ha aumentado los salarios de los grupos que utilizan computadora.

Para México no se encontró ninguna investigación que tome en cuenta la edad, la utilización de las tecnologías y su efecto en los salarios, por tanto queda abierta una línea de investigación para su posterior análisis.

\subsection{Efectos de la incorporación de tecnología por sectores y ramas económicas}

En este apartado se muestra el análisis de la incorporación de la tecnología, demanda laboral y salarios en distintos sectores y ramas económicas con producción variada y con diferentes especializaciones relativas en los factores productivos. Un elemento a considerar es que no existe una única demanda de trabajo, de manera que los efectos de la HCTs pueden divergir entre sectores de actividad y depende de la intensificación en investigación y desarrollo (I+D), capital y mano de obra calificada.

Cuando el sector se ve afectado por un cambio tecnológico, la demanda de ocupación queda sesgada a favor de los trabajadores más fácilmente adaptables a las nuevas condiciones. Como consecuencia, la proporción de estos trabajadores en el sector aumenta, lo cual repercute en un crecimiento de los salarios de este colectivo. Por su parte, los trabajadores de baja formación experimentan un descenso en la demanda de empleo, que sólo puede ser compensada con caídas en los salarios. De igual manera, la HCTS sostiene que la prima por la habilidad varía de una rama a otra debido al grado de inversión en maquinaria y equipo sofisticado y de cómputo.

El análisis de los cambios en la demanda de empleo por sectores y niveles, considerando la formación de la mano de obra, debe conducir a identificar aquéllos donde se han producido mayores transformaciones en tecnología, demanda de trabajo y salarios.

Aparte de los cambios que tienen lugar en la distribución del empleo por sectores de actividad, Oliver et al. (2001) aseveran que en cada rama productiva se pueden dar modificaciones en la composición interna de la ocupación por niveles de educación. 
Steiner y Mohr (1998) y Oliver et al. (2001) analizan este aspecto para el oeste de Alemania y Espańa, respectivamente. Utilizan diferentes tipos de información para los periodos 1983-1984 y 1995-2000, aplicando sus análisis a todos los sectores económicos. Los autores confirman que existen cambios en los salarios de los trabajadores dependiendo del sector económico donde se ubiquen. Las variaciones dentro de los sectores explican la mayor parte, asociado con una mayor presencia de empleo calificado, y el resto al cambio entre sectores.

También para España, Torres (2002), con datos de la Encuesta Industrial en el periodo 1980-1992, encontró que la composición del empleo, traducido en un aumento en la prima salarial, ha sido a favor de los trabajadores calificados, y a su vez ha tenido una evolución muy similar a la de otros países desarrollados, y esto se explica por las variaciones dentro de los sectores y no entre éstos.

La evidencia disponible sobre México sugiere que los salarios aumentan más rápidamente en los servicios de alto valor agregado, así como en algunas manufacturas de maquinaria y equipo (Ghiara y Zepeda, 1996). Esto quiere decir que la prima por la habilidad aumenta más rápido donde se concentra la inversión y donde las exportaciones son mayores.

Meza (2003) realizó un análisis de la demanda laboral para los ańos 1988 y 1998 utilizando la ENEU, y muestra que la demanda por trabajo calificado aumentó más en el sector manufacturero que en el no manufacturero, al igual que en los países más avanzados, y que estos resultados sugieren que el cambio tecnológico, más que la liberalización comercial, es la fuerza principal detrás de las variaciones laborales.

Ghiara y Zepeda (2004), en su análisis sobre la desigualdad salarial en la ciudad de Tijuana, encontraron que durante el periodo 1987-1994 crecieron los rendimientos en educación superior, asociados posiblemente a un incremento relativo en la demanda de trabajadores con estas características, pero además que este comportamiento fue diferente en las ramas manufactureras, mostrando un comportamiento más favorable en las actividades tradicionales y no precisamente en los sectores relativamente modernos, como podría ser maquinaria y equipo. Dicho comportamiento se puede explicar por el carácter maquilador de este sector en la ciudad, lo que parece cuestionar la posibilidad de una intensificación del cambio tecnológico a partir de la apertura comercial.

Según la evidencia mostrada para México en lo relativo a este apartado, se pueden destacar dos elementos: 1) existen diferencias por sectores y ramas en lo relativo al cambio técnico que se reflejan en diferencias tanto en la modificación de la estructura ocupacional entre e intrasectorial, así como en las remuneraciones relativas; y 2) el análisis sobre los rendimientos educativos por sectores y desigualdad salarial no incorpora ele- 
mentos para sostener que la causa de dicho comportamiento se atribuya a la existencia de un cambio tecnológico sesgado, aunque tampoco se puede descartar.

\subsection{Análisis regional y cambio técnico}

El aumento de la demanda relativa de trabajadores con altos niveles de educación y de habilidades, explicado por la HCTs, se asocia por la difusión de la computadora y los microprocesadores. En este sentido se debe tomar en cuenta que las empresas que tienen tecnologías en sus procesos productivos buscan un espacio donde se disponga de los recursos suficientes para operar, como el acceso a mercados, infraestructura, capital humano y aspectos institucionales en relación con el establecimiento de salarios mínimos.

Por tanto, esta racionalización de la ubicación estratégica idónea de las empresas e industrias permiten un extraordinario incremento en la flexibilidad de los procesos de producción, distribución y gestión, que se traduce en una descentralización de las distintas unidades de dichos procesos en diferentes ubicaciones espaciales; por ello, es importante la especialización del capital humano, que es la clave para que se presente el proceso de ciencia-tecnología-producción-adaptación-utilización (Castells, 1989).

La existencia de economías de aglomeración, tanto de localización como de urbanización, pueden generar que la distribución de actividades industriales y servicios no presenten una distribución homogénea en el territorio, por tanto, mientras algunas regiones pueden tener exceso de oferta, en otras la característica sería la escasez, con su consecuente resultado en salarios. Aunado a esta situación, los procesos de apertura comercial llevados a cabo en las últimas décadas puede generar alteración en las condiciones regionales preestablecidas.

Por otro lado, Rozga (1998) menciona que las nuevas tecnologías aplicadas al desarrollo regional en algunos países muestran casos exitosos. Las regiones que llegan a tener ubicaciones importantes de la invención, obtienen una enorme influencia económica y política dentro de sus naciones en el largo plazo. La capacidad regional de innovación está influenciada por el resultado previo de las invenciones y por el aprendizaje acumulativo que crea la accesibilidad de técnicas productivas con alto valor agregado, y su implementación tiene efectos diferentes según el desarrollo económico del país en que se ubique, ya que debe tenerse en cuenta que algunas naciones son generadoras y exportadoras y otras son importadoras y dependientes. 
En el caso de México, Freenstra y Hanson (1994, 1997), así como Zepeda (1997) encuentran diferencias sustanciales al interior de la región fronteriza, por ejemplo, estructura manufacturera y de servicios, montos de ingreso e incidencia de pobreza, avance educativo y otros aspectos socioeconómicos. Dichas diferencias se deben al efecto de la apertura comercial, la inversión extranjera directa (IED) y el cambio tecnológico.

Hanson (2003) utiliza datos de los censos de población de 1990 y 2000, analiza los cambios salariales desde una perspectiva regional y encuentra diferencias entre las distribuciones salariales, asimismo que esos cambios han traído consigo un mayor grado de desigualdad salarial y los factores que están detrás de dicha desigualdad son la ubicación geográfica, en particular la exposición a la globalización y no los cambios demográficos, económicos y sectoriales.

Meza (2005) hizo un análisis de 16 ciudades tomadas de la ENEU de 1988 a 1999, y sus resultados muestran que las estructuras salariales han cambiado de manera distinta entre ciudades, mientras que en Guadalajara se observan aumentos importantes en la desigualdad salarial, lo contario sucede en Monterrey o Tijuana, donde las disparidades parecen disminuir. Por otro lado, llama la atención que dicho fenómeno se concentra en el centro y sur del país, mientras que en el norte parece prevalecer una reducción de la dispersión de salarios. Sin duda resulta relevante determinar en qué medida el cambio tecnológico sesgado puede estar incidiendo como comportamiento de desigualdad salarial regional.

Barceinas y Raymond (2005) realizaron un análisis de los rendimientos educativos de 10 regiones con datos de la ENIGH de 1984 a 2002. Sus resultados muestran un aumento en la tasa de rentabilidad entre 1984 y 1994, donde algunas regiones alcanzan su máximo, para después descender ligeramente o mantenerse en los mismos niveles hasta 2002. Un hallazgo importante en esta investigación fue que la región más pobre (sur), seguida de la península, experimentaron tasas más altas, mientras que en las más desarrolladas -que corresponden al norte del país- se observan tasas bajas -en particular a partir de 1994-, por último, el Distrito Federal se encuentra por debajo de la media. Lo que demuestra que un año más de educación tiende a llevar asociado un mayor efecto en los salarios de las zonas más deprimidas que en las más prosperas, hecho que parece estar indicando la existencia de rendimientos marginales decrecientes en la dotación de factores agregados.

Castro (2007) analizó el comportamiento salarial entre 1992 y 2002 con datos de la ENEu, incluyendo 33 áreas urbanas del país. Encontró que la desigualdad salarial se incrementó hasta 1997, el origen se ubica entre grupos (educativos, ocupaciones y sexo) y al interior de los mismos. La razón se explica por diferencias en las remuneraciones a los factores pro- 
ductivos en los distintos centros urbanos del país y no tanto por la diferencia en la dotación de los mismos. Por su parte, López-Acevedo (2005), con información de la ENIGH y la ENEU para áreas urbanas y rurales en el periodo 1984-1996, muestra que la diferencia en educación explica la mayor parte de la desigualdad en los ingresos, cuya mayor tendencia se da en las áreas urbanas del país. El trabajo argumenta que tales efectos tienen explicación en el cambio tecnológico sesgado que se ha incrementado a partir de la apertura comercial.

A la luz de las evidencias mencionadas, se puede decir que durante los ańos ochenta y noventa el rendimiento educativo y la desigualdad salarial presentaron desempeños distintos regionalmente, donde el cambio tecnológico pudo ser un factor relevante en la explicación de dicho comportamiento, no obstante, al igual que en el caso de género, edad y sectores, no se ha estudiando con profundidad y no existe consenso sobre su efecto.

\section{Relación del cambio tecnológico con la educación, edad, sexo, sectores y regiones}

Si se analizan las variables relevantes del análisis de manera conjunta, se puede establecer que la tecnología ha favorecido a quienes cuentan con un mayor nivel educativo y que se encuentren calificados en la utilización de las TIC, además de que sean jóvenes y laboren en los sectores y ramas económicas que tienen mayor inversión en computadoras e investigación y desarrollo; sin embargo, en el caso del género y la ubicación geográfica, existen demandas muy especificas, como el tipo de ocupación y elementos territoriales que se deben considerar.

Un aspecto importante a destacar es que los trabajadores con más educación muestran un nivel de complementariedad mayor con la tecnología, que a su vez se refleja en el incremento de su productividad y en los salarios que perciben por su trabajo. Este rendimiento educativo se ha visto diferenciado por género, donde algunos estudios han demostrado que las mujeres que tienen mayor nivel educativo son más productivas y obtienen más rendimiento en el mercado laboral; sin embargo, se ha encontrado que existen diferencias salariales respecto a los hombres que en gran medida se atribuyen a factores de género y de discriminación. La evidencia analizada demuestra que es más común que las mujeres utilicen computadora en los centros de trabajo, en ocupaciones que no requieren estudios universitarios, y lo contrario sucede en este nivel, ya que existe una mayor complejidad en el manejo y uso de la tecnología que parece favorecer a los hombres. 
Se ha reportado que en profesiones de ingeniería, las mujeres ganan más (Rodríguez y Camberos, 2007), por lo que este comportamiento puede contribuir al cambio tecnológico sesgado.

Otro factor importante es la edad, ya que se ha encontrado que las personas más jóvenes tienen mayor capacidad para adaptarse a las nuevas tecnologías. Una posible explicación es que la población en general ha incrementado sus niveles de escolaridad, aunado a que en los últimos tiempos se han reducido los costos de las tecnologías, lo que hace más fácil obtener computadoras y aparatos electrónicos. Las mujeres jóvenes se han visto bastante favorecidas por la incorporación de la tecnología en los centros de trabajo, ya que esto hace que se simplifiquen muchas tareas y no requieran de un gran esfuerzo físico sino analítico, lo que genera que se demande mano de obra femenina.

Como se mencionó, la incorporación de la tecnología depende de los costos relativos de los factores sustituibles y la tasa de rentabilidad que obtendrían los sectores y ramas económicas en su incorporación, es por ello que el cambio tecnológico no ha llegado por igual para todos. Se ha encontrado evidencia que apunta a que el sector industrial, específicamente en la rama manufacturera, cuenta con mayor inversión en computadoras, investigación y desarrollo, y en gran medida, los cambios en la composición de la fuerza de trabajo calificada y joven para determinados giros en algunas ramas se inclinan al trabajo femenino. $\mathrm{Al}$ respecto, los giros se ubican en el sector servicios y principalmente en el área financiera, la publicidad y el comercio con un repunte en la incorporación de tecnologías.

No hay estudios sobre cambio tecnológico en el sector agropecuario, y a pesar de los esfuerzos en este sector de la economía por incorporar investigación y desarrollo con nuevas tecnologías para mejorar la capacidad productiva de los países, el efecto del cambio tecnológico es menos dinámico. Por tanto, en el contexto del efecto del cambio tecnológico sesgado esperaríamos que los sectores modernos, como el industrial y algunas ramas de servicios, sustituyeran más trabajadores menos calificados, o con un nivel bajo de instrucción, por computadoras y por trabajadores más calificados, y que en algunas ramas se inclinaran por la fuerza laboral femenina. En este proceso, los trabajadores menos calificados tendrían que ubicarse en sectores como el agropecuario y en ciertos servicios que impliquen poco requerimiento de habilidades y conocimiento tecnológico.

En el ámbito regional, los cambios en la tecnología conllevan modificaciones en la organización de la producción, que con base en mayor eficiencia en la explotación de los recursos y en los procesos de transformación industrial, replantean un reacomodo en la geografía del desarro- 
llo, en una expresión diferenciada y heterogénea en los ámbitos regional y local. Es precisamente en este contexto donde la agenda de investigación debe dirigir sus esfuerzos en el presente y futuro.

\section{Conclusiones}

Este trabajo destaca, en una primera parte, la frecuente ausencia de una explicación sobre la naturaleza de la relación entre el cambio técnico y su efecto heterogéneo en los trabajadores con diferentes características laborales, motivo por el cual se hace una presentación de la misma, destacando que la decisión de sustituir tecnología por tareas rutinarias responde a un criterio de racionalización económica.

La toma de decisiones sobre la composición tecnológica termina afectando la estructura ocupacional y/o salarial de los trabajadores, por tal motivo se puede sostener que la нстs puede ser un elemento que explique el aumento de la desigualdad entre los trabajadores; sin embargo, este mismo comportamiento puede ser compatible con otras hipótesis, por lo que se debe explorar con mucho mayor detenimiento, como queda de manifiesto en este trabajo, mediante la revisión de evidencias empíricas donde no solamente se analiza el comportamiento de la disparidad salarial y la evolución del premio a la calificación laboral o a las tareas no rutinarias, sino que debe abordar cómo el cambio técnico sesgado puede afectar a los trabajadores con características similares pero ocupados en diferentes actividades, distintas regiones, cohortes generacionales y por sexo, a partir del reconocimiento de que el cambio técnico se puede introducir a distintos ritmos entre actividades, regiones, las generaciones tienen distintas capacidades de adaptación a estas tecnologías y que la condición sexual puede no ser neutral.

La нстs se ha probado en Estados Unidos, España y el oeste de Alemania, entre otros, y se ha encontrado complementariedad entre las variables que definen el cambio tecnológico: gastos en $\mathrm{I}+\mathrm{D}$, capital físico y uso de computadoras en los centros de trabajo por los trabajadores más calificados. De ello deriva la necesidad de realizar un análisis más exhaustivo sobre los efectos del cambio tecnológico sesgado en los ámbitos nacional y regional, para identificar y conocer la repercusión en los salarios y estructura ocupacional de los trabajadores.

En el caso de México, a partir de la bibliografía revisada se ha podido constatar, por una parte, la carencia de estudios que aborden la relación entre cambio técnico y su efecto en el mercado laboral, en especial lo relativo a la desigualdad salarial y la estructura ocupacional. Por otra parte, la implementación de un nuevo modelo económico caracterizado por la apertura comercial, menor intervención del Estado en la economía, la 
flexibilidad del mercado laboral y la pérdida del poder adquisitivo de los salarios, son elementos adicionales que explican la parte que no se puede atribuir al efecto que induce el cambio técnico y la HCTs, como causas totales del crecimiento de la disparidad salarial en las décadas recientes.

$\mathrm{Al}$ analizar el efecto de las tecnologías en los salarios de diferentes grupos, como educación, edad, sexo, ramas y sectores y regiones, se observó que la mayoría de las investigaciones no utilizan propiamente variables que aproximen la нстs, sino que aseveran que sus resultados se deban a lo que predice la teoría. Por tal motivo, se destaca la necesidad de realizar más estudios sobre el tema.

Un elemento adicional a destacar es que la mayoría de los estudios sólo exploran el comportamiento de la desigualdad salarial como indicador de la posible existencia de cambio técnico sesgado; sin embargo, una fuente alternativa es analizar el comportamiento de la estructura ocupacional considerando el criterio de trabajadores calificados y no calificados (o tareas rutinarias o no rutinarias) al interior de las empresas, entre actividades, regiones y por sexo, dado que el solo indicador del rendimiento a la calificación puede no ser suficiente.

Es pertinente destacar la necesidad de que los estudios orientados a analizar los efectos del cambio tecnológico en el mercado laboral, no sólo de México sino también de países en desarrollo, partan del reconocimiento de las particularidades de cada economía y su mercado de trabajo, de tal suerte que se pueda adaptar la implementación de determinada metodología. Aunado a la existencia de mercados financieros poco desarrollados, volatilidad de los mercados, de bienes y la desregulación y flexibilidad del mercado de trabajo, como elementos que limitarían la sustitución de trabajo por tecnología, se pueden señalar los siguientes elementos relevantes de análisis en los mercados de trabajo de México y otros países en desarrollo: a) la incapacidad de los sectores formales de la economía para absorber una oferta creciente de mano de obra, lo que afecta a trabajadores con diferentes niveles de calificación y cuya consecuencia es un aumento en los niveles de desempleo y de informalidad; b) una proporción importante de la estructura industrial que en determinados casos responde a un esquema maquilador, donde la reducción de costos laborales asociado a la ventaja de localización han compuesto su razón de ser, así como un aumento de los costos laborales puede no necesariamente incentivar la introducción de tecnología, sino sólo la relocalización de los procesos productivos en otros países.

La consideración de estos elementos constituye un reto adicional en el estudio de cómo el cambio tecnológico afecta al mercado laboral, pero sin duda, representa una aproximación de este fenómeno mucho más apegado a una realidad mexicana cada vez más compleja. 


\section{Bibliografía}

Acemoglu, Daron (2002), "Technical change, Inequality, and the labor market", Journal of Economic Literature, 40, (1), American Economic Association, Pittsburgh, pp. 7-72.

Arango, Luz Gabriela (2004), "Mujeres, trabajo y tecnología en tiempos globalizados", Cuadernos CES, 5, Facultad de Ciencias Humanas, Universidad Nacional de Colombia, Bogotá, pp. 1-17.

Autor, David, Lawrence Katz y Alan Kruger (1998), "Computing inequality: Have computers changed the labor market?", Quarterly Journal of Economics, 113, Harvard University's Department of Economics, Cambridge, pp. 1169-1213.

Autor, David, Frank Levy y Richard Murnane (2003), “The Skill content of recent technological change: An Empirical exploration", Quarterly Journal of Economics, 118 (4), Harvard University's Department of Economics, Cambridge, pp. 1279-1333.

Autor, David, Lawrence Katz y Melissa Kearney (2008), “Trends in U.S. wage inequality: Revising the revisionists", The Review or Economics Statistics, 90 (2), Harvard University's Kennedy School of Government, Cambridge, pp. 300-323.

Barceinas, Fernando (1999), "Función de ingresos y rendimientos de la educación en México", Estudios Económicos, 14 (1), El Colegio de México, México, pp. 87-127.

Barceinas, Fernando (2003), "Endogeneidad y rendimientos de la educación”, Estudios Económicos, 18 (1), El Colegio de México, México, pp. 79-131.

Barceinas Fernando y José Luis Raymond (2005), "Convergencia regional y capital humano en México, de los ańos 80 al 2002”, Estudios Económicos, 20 (2), El Colegio de México, México, pp. 263-293.

Bracho, Teresa y Andrés Zamudio (1994), "Los rendimientos económicos de la escolaridad en México, 1989”, Economía Mexicana, 3 (2), Centro de Investigación y Docencia Económicas, México, pp. 345-455. 
Bowles, Samuel, Herbert Gintis y Melissa Osborne (2001), "The determinants of earnings: A behavioral approach", Journal of Economics Literature, 39 (4), American Economic Asociation, Pittsburgh, pp. $1137-1176$.

Card, David y John DiNardo (2002), "Skill-Biased technological change and rising wage inequality: Some problems and puzzles", Journal of Labor Economic, 20 (4), University of Chicago Press Journals, Illinois, pp. 733-783.

Card, David y Thomas Lemiux (2001), "Can falling supply explain the rising returns to college for younger men? A cohort-based analysis", Quarterly Journal of Economic, 116, Harvard University's Department of Economics, Cambridge, pp. 705-746.

Castells, Manuel (1989), "Nuevas tecnologías y desarrollo regional", ponencia presentada en el Seminario Internacional sobre Política Regional en Europa en los años 90, Madrid, 30 de mayo.

Castro-Lugo, David (2007), "Disparidad salarial urbana en México, 1992-2002”, Estudios Sociales, 29, Centro de Investigación en Alimentación y Desarrollo, Hermosillo, pp. 117-153.

Freenstra, Robert y Gordon Hanson (1994), "Foreign investment ourtsourcing and relative wages. Political economy of trade policy", en Enssays in honor of Jagdish Bhagwati, The MIT Press, Cambridge, pp. 89-127.

Freenstra, Robert y Gordon Hanson (1997), "Foreign direct investment and relative wages: Evidence for Mexico's maquiladoras", Journal of International Economic, 42, Elsevier, Maryland, pp. 371-393.

Ghiara, Ranjeeta y Eduardo Zepeda (1996) "Returns to education and Economic Liberalization”, Documentos de Investigación 4, Centro de Investigaciones Socioeconómicas, Universidad Autónoma de Coahuila.

Ghiara, Ranjeeta y Eduardo Zepeda (2004), "Desigualdad salarial, demanda de trabajo calificado y modernización: lecciones del caso de Tijuana, 1987-1994”, Región y Sociedad, 16 (29), El Colegio de Sonora, Hermosillo, pp. 1-43. 
Grijalva-Monteverde, Gabriela (2003), "Diferencias salariales por sexo en el sector privado en México: el caso de Hermosillo, Sonora”, El Mercado de Valores, enero, Nacional Financiera, México, pp. 13-25.

Hanson, Gordon (2003), "What has happened to wages in Mexico since nafta? Implications for hemispheric free trade", Working Paper num. 9563, National Bureau of Economic Research.

Huesca-Reynoso, Luis (2004), "La rentabilidad de la escolaridad de los hogares asalariados en México durante 1984-2000", Problemas del Desarrollo, 35 (138), Universidad Nacional Autónoma de México, México, D.F. pp. 126-154.

Huesca-Reynoso, Luis (2005), La distribución salarial del mercado de trabajo en México: un análisis de la informalidad, tesis de doctorado, Departament d'Economia Aplicada, Universidad Autónoma de Barcelona, Barcelona.

Juhn, Chinhui, Kevin Murphy y Brooks Piere (1991), “Accounting for the slowdown in black-white wage convergence", en Marvin $\mathrm{H}$. Kosters (ed.), Workers and Their Wages, American Enterprise Institute, Washington, pp. 107-143.

Juhn, Chinhui, Kevin Murphy y Brooks Piere (1993), "Wage inequality and rise in returns to skill”, Journal of Political Economy, 101 (3), University of Chicago, Illinois, pp. 410-442.

López-Acevedo, Gladys (2005), "Mexico: Evolution of earnings inequality and rates of returns to education (1988-2002)", Estudios Económicos, El Colegio de México, México, pp. 211-284.

Martínez-Jasso, Irma y Gloria Acevedo Flores (2004), "La brecha salarial en México con enfoque de género: capital humano, discriminación y selección muestral", Ciencia, 7 (1), Universidad Autónoma de Nuevo León, Monterrey, pp. 66-71.

Meza-González, Liliana (2001), "Wage inequality and the gender wage gap in Mexico", Economía Mexicana, nueva época, 10 (2), Centro de Investigación y Docencia Económicas, México, pp. 291-323.

Meza-González, Liliana (2003), "Apertura comercial y cambio tecnológico: efectos en el mercado laboral mexicano", El Trimestre Eco- 
nómico, 70 (3), 279, Fondo de Cultura Económica, México, pp. 456-505.

Meza-González, Liliana (2005), "Mercados laborales locales y desigualdad salarial en México", El Trimestre Económico, 72 (1), 285, Fondo de Cultura Económica, México, pp. 133-178.

Mincer, Jacob (1974), Schooling, experience and earning, National Bureau of Economic Research-Columbia University Prees, New York.

Oaxaca, Ronald (1973), "Male-Female differentials in urban labor markets", International Economic Review, 14 (3), University of Pensilvania, Filadelfia, pp. 696-709.

Oliver, Alonso, Raymond Josep, José Luis Bara y Héctor Sala Lorda (2001), "Necesidad de formación en el mercado de trabajo español: composición del empleo y estructura productiva", documento de trabajo 01.17, Universidad Autónoma de Barcelona, Barcelona.

Rodríguez-Pérez, Reyna Elizabeth y Mario Camberos Castro (2007), "Análisis de la discriminación de la mujer en Hermosillo, Sonora", Revista Politica y Cultura, 28, Universidad Autónoma Metropolitana-Xochimilco, México, pp. 225-256.

Romero, José y Alicia Puyana (2003), "Reforma estructural y remuneraciones a los factores: la experiencia mexicana”, documento de trabajo 5, El Colegio de México, México.

Rózga-Luter, Ryszard (1998), Desarrollo regional e innovación tecnológica: región metropolitana de Toluca como polo de innovación, Universidad Autónoma del Estado de México, Toluca.

Steiner, Viktor y Robert Mohr (1998), "Industrial change, stability of relative earning, and substitution of unskilled labor in West Germany", discussion paper, 98-22, Zew, Mannheim.

Torres, Xavier (2002), "Dispersión salarial y cambio tecnológico en la industria española”, Investigaciones Económicas, 26 (3), Fundación SEPI, Madrid, pp. 551-571. 
Urciaga-García, José (2002), "Los rendimientos privados de la educación formal en México", Comercio Exterior, 152 (4), Bancomext, México, pp. 324-330.

Urciaga-García, José y Marco Antonio Almendarez Hernández (2008), "Salarios, educación y sus rendimientos privados en la frontera norte de México. Un estudio de capital humano", Región y Sociedad, xx (41), El Colegio de Sonora, Hermosillo, pp. 33-56.

Valenzuela, Alejandro (2002), "El mercado de trabajo de Hermosillo: discriminación salarial y el nivel de escolaridad", en Sonora frente al siglo XXI, El Colegio de Sonora-Universidad de Sonora, Hermosillo, pp. 463-483.

Villarreal Peralta, Edna María (2008), "Evolución histórica de los rendimientos educativos en México: 1987-2004”, Estudios Sociales, 16 (32), Centro de Investigación y Desarrollo, Hermosillo, pp. 59-78.

Zamudio, Andrés (1995), "Rendimientos a la educación superior en México: ajuste por sesgo utilizando máxima verosimilitud", Economía Mexicana, 4 (1), Centro de Investigación y Docencia Económicas, México, pp. 69-91.

Zepeda, Eduardo (1997), "Salarios relativos y región: 1987-1993. Caso de la frontera norte de México", Estudios Sociales, 7 (14), Centro de Investigación y Desarrollo, Hermosillo, pp. 123-152.

Recibido: 20 de abril de 2009. Reenviado: 8 de febrero de 2010. Aceptado: 25 de febrero de 2010.

Luis Huesca-Reynoso. Es doctor en economía y máster en economía aplicada por la Universidad Autónoma de Barcelona. Es investigador titular y jefe del Departamento de Economía en el Centro de Investigación en Alimentación y Desarrollo, A.c. (CIAD). Obtuvo beca de repatriación por el Consejo Nacional de Ciencia y Tecnología (Conacyt) y es miembro del Sistema Nacional de Investigadores (SNI) nivel I desde el año 2006. Fue galardonado con el primer lugar en el ix Premio Nacional a la Investigación Laboral 2006 que otorga la Secretaría del Trabajo y Previsión Social y el Banco Interamericano de Desarrollo. Realiza investigación sobre temas de empleo y desarrollo económico en el CIAD y es profesor 
de cátedra en economía en el Instituto Tecnológico y de Estudios Superiores de Monterrey (ITESM), Campus Sonora-Norte. Entre sus publicaciones recientes destacan: "El mercado laboral mexicano 1992 y 2002: un análisis contrafactual de los cambios en la informalidad", Economía Mexicana, 18 (1), CidE, México, pp. 5-43 (2009); “Equidad y remesas en las regiones en México, 2000 y 2005”, Comercio Exterior, 59 (7), Bancomext, México, pp. 514-522 (2009); "Análisis regional de las remesas y sus perceptores en México, 2000 y 2005", Estudios Fronterizos, 10 (19), UABC, Mexicali, pp. 49-83 (2009); en coautoría, "Contribución de las remesas a los ingresos públicos en México", Migración y Desarrollo, 14, Red Internacional de Migración y Desarrollo, A.C., UAED, Zacatecas (en prensa).

David Castro-Lugo. Es doctor en economía y maestro en economía aplicada por la Universidad Autónoma de Barcelona; asimismo cursó otra maestría en El Colegio de la Frontera Norte. Hizo una estancia posdoctoral, apoyado por el Conacyt, en la Coordinación de Desarrollo del Centro de Investigación en Alimentación y Desarrollo (CIAD). Es profesorinvestigador titular en el Centro de Investigaciones Socioeconómicas (CISE) de la Universidad Autónoma de Coahuila. Realiza investigación principalmente en las áreas de economía laboral y economía regional. Es miembro del cuerpo académico consolidado Reestructuración regional y políticas públicas. Entre sus publicaciones más recientes destacan: "Disparidad salarial urbana en México, 1992-2002”, Estudios Sociales, 15, CIAD, México, pp. 118-153, (2007); en coautoría, "Desigualdad salarial en México: una revisión", Papeles de Población, 54, Universidad Autónoma del Estado de México, Toluca, pp. 225-264 (2007).

Reyna Elizabeth Rodríguez-Pérez. Es candidata a doctora en ciencias por el Centro de Investigación en Alimentación y Desarrollo (CIAD) y maestra en desarrollo regional titulada con mención honorífica por el CIAD. Es profesora de tiempo completo asociada "C" de la carrera de Administración y Evaluación de Proyectos en la Universidad Tecnológica de Hermosillo, Sonora. Es perfil deseable ante el Programa de Mejoramiento al Profesorado (Promep) y miembro del cuerpo académico Desarrollo regional en Sonora, reconocido ante el mismo organismo. Es evaluadora de programas académicos del Consejo de Acreditación en la Enseñanza de la Contaduría y Administración, A.c. (CACECA). Realiza investigación sobre temas de mercado laboral y desarrollo regional. Entre sus publicaciones destacan: en coautoría, "Mercado laboral, inequidad salarial y cambio tecnológico regional”, Frontera Norte, 23 (45), Colegio de la Frontera Norte, Tijuana (en prensa); en coautoría, "Salarios y cali- 
ficación laboral en México", Problemas del Desarrollo, 39 (154), unam, México, pp. 61-86 (2008), en coautoría, "Análisis de la discriminación salarial de la mujer en Hermosillo, Sonora", Política y Cultura, 28, uAM, México, pp. 225-256 (2007). 